\title{
Estrategias de comunicación en campañas electorales: El caso de la contienda presidencial de Estados Unidos 2008*
}

\author{
Andrés Valdez Zepeda y Delia A. Huerta Franco (Universidad de Guadalajara) \\ Recibido: 08/09/08 \\ Aprobado: 15/10/08
}

RESUMEN: En el artículo se hace un análisis de las estrategias de comunicación que utilizó Barack Obama para ganar la nominación como candidato del Partido Demócrata a la presidencia de Estados Unidos y las que se están impulsando como parte de la campaña constitucional en el 2008, tanto por los republicanos como por los demócratas. Se concluye que Obama ganó la nominación por ser el precandidato que mejor se supo comunicar y conectar con los electores. Su alta competencia comunicativa, así como sus estrategias de comunicación, organización y movilización electoral le permitieron derrotar a la otrora poderosa Hillary Clinton.

Palabras clave: Estrategias de comunicación - campañas electorales competencias comunicativas y candidato del cambio - campañas negativas.

\section{Comunication strategies for electoral campaigns: The 2008 United States presidencial contest}

SUMMARY: The essay analyzes the communication strategies used by Barack Obama in order to win the democratic nomination to the presidency of the Unites States and the strategies used by republicans and democrats in the constitutional election in 2008. It is concluded that Obama won the presidential nomination because he was the candidate more competitive in communicate with voters. His elevated communication competences, his excellent organization and mobilization strategies of voters and the creativity of his campaign allowed him to defeat to Hillary Clinton' electoral machinery.

Key words: Comunication strategies - election campaigns - communicative competence and change candidate - negative campaigns.

* El artículo fue escrito antes de que se realicen las elecciones presidenciales de Estados Unidos, el 4 de noviembre del 2008, en las que Obama resultó ganador. [N. del E.] 


\section{Introducción}

$\mathrm{T}$ oda campaña electoral implica un ejercicio de comunicación persuasiva que realizan, previa a la jornada electoral, partidos, candidatos, equipos de campaña y simpatizantes, con el fin, por un lado, de obtener el voto de los electores y, por el otro, de evitar que sus opositores ganen la contienda. Es decir, toda campaña electoral es, en esencia, una actividad de comunicación orientada a construir mayorías electorales y acceder, en consecuencia, a una posición de representación pública.

En estas campañas, las estrategias de comunicación que impulsan los candidatos, sus partidos y simpatizantes juegan un papel decisivo, ya que las campañas electorales se ganan o se pierden en el nivel estratégico y táctico. Esto es, las estrategias de comunicación pueden determinar el éxito o el fracaso de una contienda electoral, ya que también hay campañas equívocas e insípidas que no logran una conexión emocional ni un efecto persuasivo hacia los electores.

Las estrategias de comunicación de una campaña son acciones deliberadas (generalmente creativas e inteligentes), que realizan los candidatos, partidos, equipos de campaña y simpatizantes, orientadas a crear y mantener ventajas competitivas y comunicar mensajes emotivos a grupos específicos de electores, usando distintos medios, con el objetivo de per- suadir, motivar, movilizar y lograr el respaldo y el voto de los ciudadanos.

Toda estrategia de comunicación debe, entre otras de sus características, formularse con anticipación a su implementación, buscar alcanzar un objetivo determinado, estar dirigida hacia un target específico, generar un impacto en el comportamiento o conducta del elector, contrarrestar a los grupos o candidatos opositores $y$, sobre todo, movilizar los sentimientos y emociones de la gente para lograr el voto a favor de sus impulsores.

Una de las campañas internas que sobresalió en el contexto internacional en materia de estrategias de comunicación política es la que se desarrolló en Estados Unidos por parte de Barack Obama, desde finales del 2007 y hasta mediados del 2008.

En el presente escrito se describen y analizan las estrategias de comunicación que utilizó Barack Obama para obtener la postulación como candidato del Partido Demócrata a la presidencia de Estados Unidos en la elección primaria, derrotando a Hillary Clinton e imponiéndose como el candidato favorito para ganar la contienda constitucional de noviembre del 2008.

\section{Las campañas electorales}

Las campañas electorales son, hoy día, procesos intensos de investigación, comunicación, proselitismo, organización, movilización y cuidado 
y defensa del voto que realizan partidos, candidatos, equipos de campaña y sus simpatizantes con el objetivo, por un lado, de ganar una elección y, por el otro, de evitar que la competencia logre obtener una mayoría de votos. Es decir, toda campaña implica la conformación de un frente de atracción de sufragios para la causa buscada y un frente de repulsión de votos para los adversarios.

La historia de las campañas electorales es tan antigua como lo es la democracia ateniense del siglo $\mathrm{V}$ a. C., en la antigüedad clásica. Sin embargo, las campañas electorales modernas, tal y como las conocemos actualmente, tienen una historia más reciente. La primera campaña política fue

[...] la que llevó a cabo el primer ministro británico William Gladstone, entre 1876 y los 1880. Esta campaña (conocida como la Campaña de Midlothian, por la ciudad escocesa) consistió en una serie de discursos, algunos de más de cinco horas, sobre la política exterior británica en relación a las atrocidades que cometía el Imperio otomano contra los búlgaros. ${ }^{1}$
En Estados Unidos la primera campaña electoral se realizó en $1793 .{ }^{2}$ Esta campaña se caracterizó por utilizar estrategias de comunicación y contacto más directas entre los candidatos y sus electores. Sin embargo, con el avance de la tecnología y los medios de comunicación, principalmente electrónicos, así como con la instauración del voto universal (masificación del sufragio) las campañas electorales adquirieron un perfil preponderantemente mediático. ${ }^{3}$

Hoy día, las campañas se han transformado en ejercicios sofisticados y modernos en los que se involucran e invierten grandes sumas de dinero, tiempo y recursos humanos para tratar de alcanzar o conservar el poder. Estas campañas son ejercicios proselitistas inteligentes, en las que los hombres e institutos políticos más astutos y capaces logran conquistar la mente y el corazón de los electores, para ganar el voto a su favor. Estos esfuerzos proselitistas son prácticas comunes en todos los países con sistemas políticos democráticos, quienes han institucionalizado las elecciones como una forma de disputa civilizada

1 Véase "Campañas electorales". [en línea] Wikipedia. <http://es.wikipedia.org/wiki/Campa\%C3\%B1a_pol\%C3\%ADtica>.

2 George Washington presidió en 1787 la Convención de Filadelfia, que esbozó la Constitución de los Estados Unidos de América, y en 1789 fue elegido, de manera unánime, como el primer presidente de Estados Unidos.

3 La radio se utilizó por primera vez en la campaña de 1924, y la televisión surgió en 1952 como el vehículo principal para comunicarse con los votantes. A partir de este año, la televisión se impuso como medio preponderante en las campañas electorales. 
del poder entre diferentes grupos sociales y formaciones partidistas.

En un contexto de mayor competencia inter e intrapartidista, y con el desarrollo de las nuevas tecnologías de la información, principalmente la internet, las campañas electorales han adoptado, también, un formato digital, utilizando la web y el correo electrónico como instrumentos para recaudar fondos, persuadir, organizar y movilizar a los votantes. Es decir, hoy día las campañas electorales han adquirido un perfil tanto mediático como digital, utilizando las nuevas tecnologías de la información y las comunicaciones como medios para ganar votos y ocupar espacios de representación pública.

Esto es lo que ha pasado no solo en Europa y América Latina sino también en Estados Unidos, el que ha sobresalido a escala mundial como país van-guardista en materia de innovación y creatividad en el tema de las campañas electorales. ${ }^{4}$

\section{Las precampañas en Estados Unidos}

Como parte de su sistema electoral, los dos más grandes y representativos partidos de la Unión Americana (Demócrata y Republicano) han institucionalizado un procedimiento de competencia interna para postular a sus candidatos a la Presidencia de la República, llamado popularmente como "elecciones primarias". Su historia se remonta a los inicios del siglo $\mathrm{XX}$, cuando Theodore Roosevelt fue electo, mediante votación abierta, como candidato del Partido Progresista a la presidencia en 1901.

Hoy día, estas elecciones inician en enero del año electoral, con los comicios estatales en Iowa $\mathrm{y}$, concluyen, generalmente, en agosto con la realización de las convenciones nacionales de los dos partidos, en las que los delegados electos en los diferentes estados y los superdelegados nominan oficialmente a su candidato presidencial.

Las últimas precampañas, tanto del Partido Republicano como del Partido Demócrata, concluyeron realmente en los meses de marzo y junio del 2008, respectivamente, cuando John McCain ganó la mayoría de votos necesarios para ser postulado como candidato de los republicanos y Barack Obama como abanderado de los demócratas.

4 Estados Unidos fue el primer país donde se desarrollaron debates en televisión entre los candidatos presidenciales John F. Kennedy y Richard Nixon, en 1960. En el 2008, los debates entre los candidatos se han realizado no solo en televisión sino contando, también, con el apoyo de internet (YouTube), lo cual permite que los ciudadanos realicen preguntas y cuestionamientos de manera directa, por medio de videos, a los aspirantes a ocupar la máxima magistratura. 
Esta última precampaña ha sido calificada como una contienda histórica, no solo porque compitieron en el lado demócrata, por primera vez, una mujer (Hillary Clinton) y un afroamericano (Obama), sino también porque fue una elección primaria que logró la participación y entusiasmo de millones de estadounidenses, siendo capaz de arrancar a millones de votantes del desencanto y la apatía.

\section{Las estrategias de comunicación de Obama}

Ganar una elección es cuestión de estrategia política. Persuadir y movilizar a los electores implica, además, una gran capacidad de comunicación, la que se logra a través de un adecuado trazo estratégico. De acuerdo con Rafael Alberto Pérez (2008) la estrategia de comunicación es el conjunto de decisiones sobre comunicación (tácticas) preparadas de antemano por el comunicador y su equipo para el logro de los objetivos asignados, teniendo en cuenta todas las posibles reacciones de los otros jugadores (competidores-cooperadores), de sus audiencias (públicos objetivo) o de la naturaleza (cambios de las tendencias del entorno).

En la campaña interna del Partido Demócrata, Obama tuvo la habilidad para diseñar y poner en operación un adecuado trazo estratégico en materia de comunicación electoral, que finalmente le resultó favorable para alcan- zar sus objetivos políticos. De esta forma, Obama logró imponerse en las elecciones primarias gracias a lo certero y la contundencia de sus estrategias de comunicación. Supo comunicarse mejor con los votantes, persuadirlos, organizarlos y movilizarlos el día de las votaciones. Supo, además, conseguir no solo su voto, sino también el apoyo financiero necesario para fondear su campaña.

La estrategia de comunicación de Obama se centró en presentar al precandidato como un político diferente, que asegura cambiar, para bien, el caduco sistema político y gubernamental de los Estados Unidos. Un hombre de lucha, producto de la cultura del esfuerzo y con la energía y la visión necesaria para cambiar, de una vez y para siempre, la actual situación predominante, caracterizada por la recesión económica, el hartazgo de la gente y la guerra. En este sentido, la estrategia de presentar a Obama en forma estereotipada como el candidato del cambio fue exitosa, ya que ningún mulato o afroamericano ha ocupado nunca el puesto de presidente de la nación más poderosa del mundo.

El estratega en jefe de la campaña de Obama, David Axelrod, elaboró el mensaje central de campaña

[...] a partir de la propia biografía del candidato, de su personalidad contradictoria y su trayectoria, caracterizada por el esfuerzo, el compromiso, la determinación y la superación, pro- 
yectando como un relato épico la autenticidad de la historia de aprendizaje de la vida de Barack Obama. ${ }^{5}$

De esta forma, el senador por Illinois fue presentado a los votantes como el símbolo de la esperanza y el cambio, que muchos electores anhelan para hacer suyo el sueño americano de bienestar y prosperidad, contrario a los intereses de la vieja y corrupta clase política, encumbrada en las estructuras de poder en Washington. Alguien con estatura similar o mayor a Martin Luther King, John F. Kennedy o Theodore Roosevelt, representantes del simbolismo de la política e historia norteamericana.

Sus asesores de campaña lo han sabido vender como el hombre global en la era de la mundialización, que es capaz de dialogar y generar los consensos necesarios, incluso con los principales enemigos de Estados Unidos. Un hombre de bien, pacifista y con el talante necesario para recuperar el liderazgo mundial a través de la diplomacia y no por la violencia y la imposición.

De esta forma, la estrategia de comunicación de Obama se ha centrado en el principio de la bisagra, el cual se articula en dos grandes brazos: la campaña mediática y la campaña de tierra. La primera a través de los medios de comunicación, principalmente la televisión y la radio, y la segunda, a través de la internet y el contacto directo con los votantes. La campaña mediática, le ha permitido visibilidad, presencia y comunicación abierta hacia las masas del mensaje central de la campaña, mientras que la campaña de tierra le ha posibilitado la organización y el contacto directo con los votantes, además de la recolección de fondos para financiar su campaña.

De hecho, la campaña de Obama no solo ha sido una de las más eficaces en la comunicación del mensaje del "cambio que la gente quiere y que el país necesita" a través de la internet, sino también la mejor en la historia mundial en lo que se refiere a la recolección de fondos (más de 296 millones de dólares) para el financiamiento de las actividades proselitistas.

Esta estrategia de comunicación de Obama ha sido cuidada y pulida con esmero, tanto en materia de los mensajes que se emitieron, el diseño de la página web, como el logo de campaña. De acuerdo con Gutiérrez Rubí,

[...] el logo de Obama fue creado por la compañía Sender LLC de Chicago, una conocida agencia local de diseño de identidades corporativas y páginas

5 Véase Gutiérrez-Rubí, Antoni. “Claves de la comunicación política de Obama” [en línea]. <http://www.gutierrez-rubi.es/?p=513>. 
web. Una de las características más interesantes del logo de Obama es su capacidad para declinarse, para transformarse, para adquirir capas de personalización y diferenciación según cada público. Recuerda al juego gráfico y estético que utiliza Google con la fuerte base de reconocimiento tipográfico de su marca y los cambios que vemos en la página principal en función del calendario, del Día de San Valentín, o de cualquier otra referencia que pueda asociar la marca Google a un hecho "global". Lo mismo sucede con el logo de Obama y sus múltiples versiones: "Kids for Obama", "Veterans for Obama" o "Women for Obama". 6

Todos ellos, agrega Gutiérrez Rubí, con el anclaje visual de la letra " $\mathrm{O}$ ", la primera letra de su apellido, que diseñada con los colores de la bandera norteamericana (azul, blanco y rojo) configura su símbolo político de referencia. La misma "O" que forma parte de la palabra HOPE. La misma " $\mathrm{O}$ " que se mantiene en la metamorfosis conceptual y gráfica que se ve en el vídeo YES, WE CAN, al transformar HOPE en VOTE manteniendo la " $\mathrm{O}$ " cohesionadora, raíz común de una sólida arquitectura conceptual: $O$, HOPE, VOTE, Obama. ${ }^{7}$

Obama, además, ha creado una poderosa maquinaria de inteligencia y contrainteligencia, capaz de responder de inmediato, a través de las nuevas tecnologías de la información, a los diferentes ataques y rumores impulsados por sus adversarios. ${ }^{8} \mathrm{De}$ esta forma, por ejemplo, Obama dio una respuesta rápida y contundente a los ataques de sus adversarios. Cuando sus contrincantes lo presentaron como "un riesgo para el país" Obama cuestionó la ética de la ex primera dama, así como su experiencia real en la política exterior. Cuando Hillary Clinton señaló que Obama era un inexperto, lo que ponía en peligro a los americanos y que les iba a ser perder el empleo, el senador contestó: "Hillary es un monstruo, está dispuesta a hacer lo que sea para ganar".

En el campo digital, Obama también tomó la delantera. De acuerdo con Mario Accurso, Obama les ha ganado, también, a sus adversarios, tanto a Clinton como a McCain, la guerra en el mundo virtual. Por ejemplo, para el caso de su nuevo contrincante, para el 11 de junio del 2008,

6 Ibídem.

7 Ibídem.

8 Los ataques en contra de Obama han sido distintos. Los más comunes apuntan que el senador por Illinois "es un musulmán, que es un personaje débil, un político inexperto, ingenuo, sin experiencia en asuntos de política exterior ni en temas militares, que no está a la altura del desafío que reclaman los actuales tiempos". 
Obama tenía 390.729 amigos en MySpace, frente a 53.259 de McCain. En la red social Facebook Obama registró para esa fecha 928.905 partidarios, mientras que el candidato republicano tan solo 139.749 seguidores. ${ }^{9}$ En la campaña interna, Obama dejó muy atrás a Clinton, quien prefirió las estrategias mediáticas tradicionales y no supo articular una estrategia digital que le permitiera llegar a millones de electores estadounidenses que poseen una cuenta de internet.

Obama ha sido exitoso, además, porque en su lenguaje y en su retórica por excelencia ha acudido a un formato para-religioso, propio de un gran predicador, cargado de mensajes de esperanza, devoción y misticismo. Sus contrincantes, por su parte, han acudido a una retórica habitual propia de los políticos tradicionales.

El triunfo de Obama se explica, también, porque fue el único de los precandidatos demócratas en hacer que la gente se organizara e hiciera campaña por él, logrando, de esta forma, cautivar el corazón de las masas, movilizar sus sentimientos y establecer un contacto emocional con millones de estadounidenses. Más que la campaña de Obama, esta fue una campaña de la gente. De hecho, la mejor campaña que un político puede tener es aquella que logra sensibilizar y excitar a los votantes, aquella que logra tocar las cuerdas sensibles de los electores y establecer una conexión emocional con los ciudadanos. Esto es lo que hicieron Obama y su equipo de campaña, y lo hicieron de forma excelsa.

Sus habilidades discursivas, su encanto personal, su juventud, su imagen de hombre de familia y su religiosidad, aunada a su gran capacidad e inteligencia organizativa posibilitaron que ganara la nominación del Partido Demócrata para enfrentar en noviembre del 2008 a John McCain, candidato del Partido Republicano.

Uno de los aciertos más importantes de Obama fue mantener el lema central de la campaña durante los cinco meses de la contienda interna, evitando cambiarlo como sucedió en varias ocasiones con Hillary Clinton. ${ }^{10}$ De esta manera, el cambio (“El cambio en el que podemos creer") se convirtió no solo en el mensaje central de Obama, sino también en la esperanza de millones de norteamericanos cansados de un presidente belicoso, autoritario, mentiroso y poco sensible a los problemas más sentidos de la gente.

9 Véase Accurso, Mauro, editor de NP y periodista de Clarín Zonal La Matanza [en línea]. $<$ http://www.nopublicable.com.ar/staff $>$.

10 Hillary usó diferentes lemas en la campaña: inició con "Una candidata con experiencia". Al final utilizó el lema "Cada voto cuenta". 
Obama supo hacer, además, una crítica feroz y verosímil hacia el stablishment de la política norteamericana. Supo aprovechar el hartazgo de la gente con el sistema político del país y logró venderse, desde el inicio de la contienda, como un candidato ganador, como el candidato del cambio y la esperanza, el único que puede limpiar la obscena política nacional. ${ }^{11}$

Su mensaje de campaña y su plataforma electoral abarcaron un sinnúmero de temas y propuestas, siempre de carácter propositivo, entre los que sobresalen los temas económicos, las relaciones internacionales, la educación y la salud, entre otros. En el caso de la guerra en Irak, Obama ha propuesto una salida de las tropas de los Estados Unidos, en forma cuidadosa y responsable.

\section{La campaña constitucional}

Una vez lograda la nominación de Obama, se intensificaron las campañas de los republicanos con el objetivo de desprestigiarlo, acusándolo, entre otras cosas, de no ser nacionalista, de ser musulmán, de ser una persona desconocida y, sobre todo, de no tener la experiencia para ocupar el puesto de Presidente de la República y comandante en jefe de las Fuerzas Armadas de Estados Unidos.
De esta forma, la estrategia de comunicación de los republicanos estuvo centrada en atacar, denostar y generar duda sobre las "credenciales" de Obama, cuestionando su poca experiencia en asuntos de política exterior $\mathrm{y}$, sobre todo, atacándolo por su egocentrismo. La misma Sarah Palin, candidata republicana a la vicepresidencia, señaló en la convención del Partido Republicano que "hay quienes utilizan el cambio para promover su carrera, mientras que hay otros que ponen su carrera para promover el cambio". Por ejemplo, se utilizaron, además, por los republicanos, la imagen y la voz de Hillary Clinton y los señalamientos críticos que realizó durante la campaña interna, en forma de spot publicitario, para atacar a Obama.

Por el lado de los demócratas, la estrategia de comunicación se centró en ligar a John McCain con los Bush, sus errores, insuficiencias, fracasos y abusos. De esta forma, se publicitó que el nominado por el Partido Republicano representaba la continuidad de Bush en la presidencia de Estados Unidos (guerra, crisis económica, alza de precios en alimentos y combustibles y déficit fiscal, entre otros). Asimismo, se criticó severamente, entre otras cosas, la poca experiencia de Palin en asuntos de política exterior,

11 Obama tuvo la habilidad de presentarse como el Mr. Clean de Washington, el único candidato capaz de cambiar las cosas en ese país. 
así como el embarazo de su hija Bristol, de 17 años, y su postura en perforar el territorio de Alaska en búsqueda de hidrocarburos por parte de las empresas petroleras.

Como parte de las contraestrategias de los demócratas, se estableció, además, un equipo de respuesta rápida vía internet para tratar de contrarrestar, de manera inmediata, todos los ataques, rumores y señalamientos negativos e injuriosos que los republicanos estaban impulsando, sea por internet, vía mediática o a través del contacto directo con los electores, para dañar la credibilidad y el avance de Obama hacia la presidencia.

El mismo candidato a vicepresidente por los demócratas, Joseph Biden, se sumó a la estrategia de ligar la fórmula McCain-Palin con la idea de continuidad del desastre de los Bush, aprovechando la baja popularidad del presidente y la difícil situación económica que viven los estadounidenses.

\section{Comentarios finales}

Las estrategias son definidas como las acciones inteligentes desarrolladas por una organización con el objetivo de superar a los adversarios. Son formas creativas de lograr ventajas competitivas y así alcanzar el liderazgo. En la política, la estrategia puede determinar el éxito o el fracaso de una elección. Una campaña sin estrategias o con estrategias inadecuadas o desar- ticuladas seguramente será una perdedora. Por el contrario, una campaña con estrategias adecuadas será, indudablemente, una campaña ganadora.

Obama ganó la nominación del Partido Demócrata porque supo comunicarse mejor con los votantes, les tocó las cuerdas sensibles y les habló del cambio que la gente espera y desea. Su historia personal, su color de piel y sus estrategias de comunicación hicieron posible superar la apatía y el desgano tradicional de millones de estadounidenses, quienes ven en este personaje la posibilidad de materializar un cambio verdadero en beneficio de la gente y no de las corporaciones.

A través de internet, Obama logró comunicarse con millones de electores, siendo capaz de conseguir no solo su voto para ganar la nominación sino también el apoyo económico para financiar su campaña. A través de las diferentes giras y presentaciones públicas, a lo largo y ancho del territorio estadounidense, logró, también, un contacto más directo con los ciudadanos. Por medio de la televisión alcanzó una alta visibilidad mediática y presencia permanente en casi la totalidad de los medios de comunicación.

De esta forma, Obama utilizó tanto el modelo broadcast como el modelo participativo, centrado en la organización de base y el contacto personal con los electores. Como gran estratega, supo definir y transmitir con claridad y autenticidad quién era y qué 
ofrecía, siendo astuto para enviar el mensaje que los votantes querían escuchar. En este sentido, Obama mostró una mejor competencia comunicativa que Clinton, impulsando una campaña altamente significativa $y$ tomando en cuenta el momento y la coyuntura que está viviendo la Unión Americana.

Obama tuvo la habilidad, además, de evitar en lo posible los temas ríspidos que dividen a los norteamericanos, como la discusión sobre el aborto, la legalización de las drogas y la oficialización de los matrimonios homosexuales. Al contrario, supo plantear a los votantes los temas que los unifican, como el de la familia, el trabajo, la economía, los problemas sociales y el rechazo a la guerra.

Hoy día, Obama es el candidato al que hay que vencer por parte de los republicanos, ${ }^{12}$ quien ha sabido posesionarse como el hombre que representa el cambio que la gente quiere y el país necesita. Un presidente que acabe con los abusos del sistema político y genere las condiciones para un mejor desarrollo económico, social y cultural. Un hombre que desde su co- lor de piel y su historia personal representa el cambio que los estadounidenses sueñan y que posibilite poner fin al gobierno conservador de George W. Bush, considerado en diferentes encuestas de opinión, como la peor administración y gobierno que ha tenido la Unión Americana en los últimos años.

Sin embargo, la estrategia de comunicación de los republicanos, ya como parte de la campaña constitucional hacia la Presidencia de la República, se ha centrado en atacar, denigrar y generar duda entre los votantes sobre los verdaderos valores, la fe, la experiencia y los ideales e intenciones de Obama, a quien dibujan como un personaje desconocido, inexperto, izquierdista, radical y, sobre todo, poco patriota, quien no tiene los antecedentes ni la experiencia ni los valores para ser electo como presidente de este país. Estas campañas negativas, así como el factor racial, aún no superado en muchos electores, han generado una especie de empate técnico en las preferencias electorales, de tal forma que la moneda está aún en el aire en está histórica contienda electoral.

12 Ya en su campaña constitucional, Obama está utilizando la estrategia de la triangulación para tratar de ganarle votos del sector conservador a los republicanos, moderando su discurso y hablándoles sobre temas propios de la agenda conservadora. En este orden de ideas, deben entenderse, por ejemplo, sus señalamientos en el sentido de que "defiende la pena de muerte en determinados casos" y que "está a favor de que la gente pueda portar armas", lo que es una bandera propiamente de los republicanos. 


\section{Bibliografía}

Accurso, Mauro [en línea]. <http:// www.nopublicable.com.ar/staff>, 11 de junio del 2008. [Consulta: 3 de julio del 2008.]

"Campañas electorales" [en línea]. Wikipedia. <http://es.wikipedia.org/wiki/Campa\%C3\%B1a pol\%C3\%ADtica>. [Consulta: 1 de julio del 2008.]
Gutiérrez-Rubí, Antoni. “Claves de la comunicación política de Obama" [en línea]. <http://www. gutierrez-rubi.es/?p=513>, 7 de marzo del 2008. [Consulta: 3 de julio del 2008.]

PÉREZ, Rafael Alberto. Estrategias de comunicación. $4 .^{\mathrm{a}}{ }^{\text {edición. Barce- }}$ lona: Ariel, 2008. 